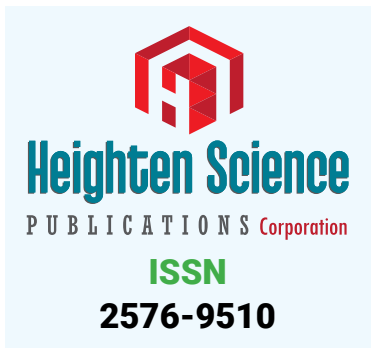

*Address for Correspondence: Ali AM, Faculty of Veterinary Medicine, University of Khartoum, P.O. Box 32, Khartoum North, Sudan, Email: awadia_mosa@hotmail.com

Submitted: 07 August 2017

Approved: 05 September 2017

Published: 06 September 2017

Copyright: @ 2017 Osman TM, et al. This is an open access article distributed under the Creative Commons Attribution License, which permits unrestricted use, distribution, and reproduction in any medium, provided the original work is properly cited.
Research Article

\section{Investigation on Theileria lestoquardi infection among sheep and goats in Nyala, South Darfur State, Sudan}

\author{
Osman TM${ }^{1}$, Ali $\mathrm{AM}^{2 *}$, Hussein $\mathrm{MO}^{3}$, El Ghali $\mathrm{A}^{4}$ and Salih $\mathrm{DA}^{4}$ \\ ${ }^{1}$ Ministry of Animal Resources, South Darfur State, Nyala, Sudan \\ ${ }^{2}$ Faculty of Veterinary Medicine, University of Khartoum, P.O. Box 32, Khartoum North, Sudan \\ ${ }^{3}$ Central Laboratory, Khartoum, Sudan \\ ${ }^{4}$ Central Veterinary Research Laboratory, P.O. Box 8067, Al amarat, Khartoum, Sudan
}

\section{Abstract}

This study was conducted in Nyala, South Darfur State, Sudan during August-September 2015 to study the seroprevalence of ovine and caprine theileriosis and to identify the tick infesting sheep and goats. For this purpose, total of 150 samples (ticks, blood smear and sera) were collected from sheep ( $n=61)$ and goats $(n=89)$ of different age groups, breed and both sex. Three age groups were included: less than one-year-old, onetwo years-old and more than two-year-old. Two diagnostic techniques were used, blood smears, and indirect fluorescent antibody test (IFAT). Out of 150 samples, $9(6 \%)$ were positive for Theileria spp. piroplasms in blood smears, and 81 (54\%) were positive for Theileria lestoquardi antibodies. Out of 61 sheep, 3 (4.9\%) were positive for Theileria spp. piroplasms, and $25(41 \%)$ were positive for $T$. lestoquardi antibodies. Out of 89 goats, $6(6.7 \%)$ were positive for Theileria spp. piroplasms, and $56(62.9 \%)$ were positive for $T$. lestoquardi antibodies. The highest prevalence of $T$. lestoquardi was recorded among sheep and goats more than two-year-old. Two genera and 4 species of ticks were infested sheep and goats. These included Rhipicephalus evertsi evertsi, R. s. sanguineus, Amblyomma variegatum and A. lepidum. The study concluded that the malignant ovine theileriosis is endemic in Nyala town.

\section{Introduction}

Tick-borne diseases (TBDs) are one of the most important constraints to livestock production in developing countries [1]. They are responsible for high morbidity and mortality resulting in decreased production of meat, milk and other livestock byproducts together with the loss of draught power. Theileria are protozoan parasites infecting wild and domestic animals throughout much of the world [2].

Malignant ovine theileriosis (MOT) is a parasitic disease of sheep and goat, caused by Theileria lestoquardi and transmitted by Hyalomma species [3]. MOT occurs in Southeastern Europe and North Africa, the near and Middle East, India, China and Southern USSR [2]. It was first described in Sudan by Mason [4], then reported in Khartoum State [5] and northern part of the country [6] and distributed in the main grazing areas [7]. Taha et al. [8], had documented an outbreak of MOT among goats in Northern Sudan, during which 16 out of 22 (72.7\%) goats were died showing clinical signs of malignant caprine theileriosis as well as in the postmortem findings, the infection was detected microscopically and confirmed serologically by IFA test and molecularly by PCR.

Nyala is one of the richest towns concerning of livestock population. Sheep and goats are among the major economically important in town estimated about 3.6 million sheep, 2.9 million goats [9]. It is playing an important role in the livelihood of resourcepoor farmers. They provide their owners with a vast range of products and services such as meat, milk and hide. 
This study was conducted in Nyala due to lack of data regarding ovine and caprine theileriosis in the region. The objective of this study was to investigate the occurrence of Theileria lestoquardi in Nyala, South Darfur State and to identify the tick infesting sheep and goats which could act as a potential vectors for the parasite.

\section{Materials and Methods}

Samples were collected during August and September 2015 from 150 animals (89 goats, 61sheep) from six locations in Nyala, South Darfur State (Elserife, Slaughter house, Elmauashi, Mogf-genana, Elshabae and Mosy) (Figure 1). The breeds of sheep and goats were local (desert breeds) and crosses (Saanen and Guprus). Three age groups were included: less than one-year-old, one-year-two year-old and more than two-year-old.

Total body ticks were collected from the predilection sites of the 150 sheep and goats. The ticks of each animal were separately preserved in vials containing $70 \%$ ethanol. Thin blood smears were made from ear veins using a sterile needle and fixed by absolute methanol. Five $\mathrm{ml}$ of whole blood were collected from the jugular vein of each animal using sterile syringes. The blood was allowed to clot in the fridge $\left(+4^{\circ} \mathrm{C}\right)$ overnight and, then, centrifuged at $2000 \mathrm{rpm}$ for 10 minutes. Serum was, removed by a separate Pasteur pipette for each sample and transferred to eppendorf labelled tubes. The serum samples were, kept at $-20^{\circ} \mathrm{C}$ until used. Blood smears, sera and ticks were labeled indicating sample number, animal breed, locality, date of collection, and sex.

The ticks were identified under a dissecting microscope according to the methods described by Hoogstraal, and Walker et al. [10,11]. The identified ticks were recorded according to sex and species. Slides were stained by Giemsa stain and examined under100 X oil immersion objective using a binocular microscope (Olympus, Japan) for the presence of Theileria spp. piroplasms. Indirect fluorescent antibody (IFA) test was carried out to detect $T$. lestoquardi antibodies. Techniques used were essentially adopted from $[12,13]$. In brief, the schizonts antigen was prepared from passage 48 of T. lestoquardi schizonts culture (CVRL Soba). The antigen suspension was applied onto the IFA slides (Immune floracin antibody slide, Germany). The slides were individual wrapped in tissue paper and packed in aluminum foil with 5 slides in each packet and stored at $20^{\circ} \mathrm{C}$ until used.

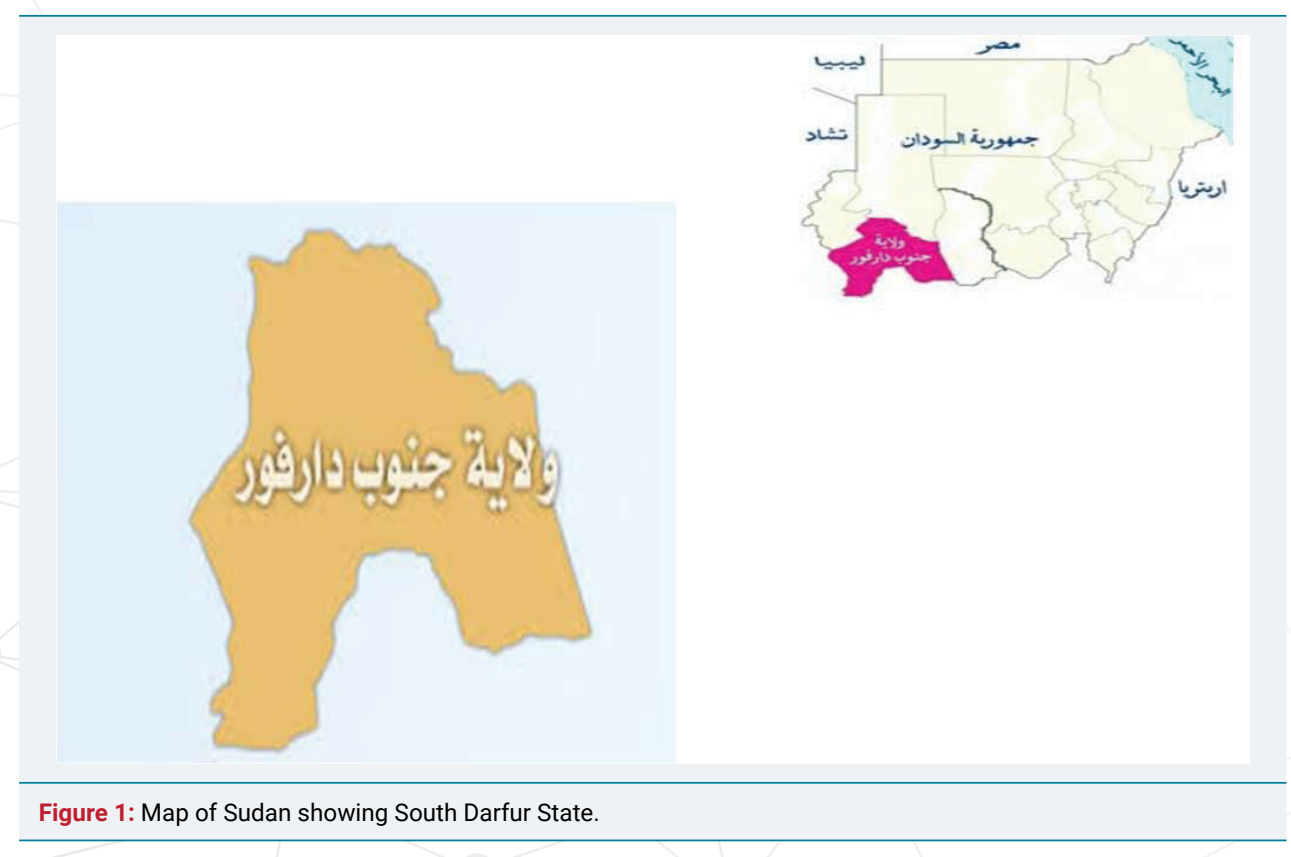


An aliquot $(0.1 \mathrm{ml})$ of the conjugate (anti-sheep immune gamma globulin $\operatorname{IgG}$ ) was thawed at room temperature and diluted $1 / 10$ in PBS. Evans blue $(50 \mu \mathrm{l})$ at a concentration of $0.1 \%$ was added to the conjugate as a counter stain to improve the definition of specific fluorescence.

Positive control $(\mathrm{C}+\mathrm{ve})$ was obtained from sheep experimentally infected with $T$. lestoquardi after 3 Months. While negative control (C-ve) was obtained from the same sheep before infection with T. lestoquardi, Central Veterinary Research Laboratories (Soba). Control sera were diluted directly to $1 / 80$.

Test sera collected from the field were allowed to thaw at room temperature, and dilution in $1 / 80 \mathrm{PBS}$. Schizont antigen slides were allowed thaw at $4^{\circ} \mathrm{C}$ for 30 minutes, placed at room temperature for another 30 minutes before they were unwrapped and labeled with location and animal numbers.

The antigen slides were thawed and labeled then placed in Petri dishes on a moist filter paper. The test sera added to the wells and were put in a plastic box with moist filter paper and incubated at $37^{\circ} \mathrm{C}$ for 30 minutes to allow antigen/antibody reaction take place. They were then dipped with PBS in staining jar for 30 minutes to remove excess sera, the buffer being renewed after the first 15 minutes. The slides were replaced in Petri dishes with a moist filter paper, and $10 \mu \mathrm{l}$ of the diluted conjugate was applied to each well of the slides. Then they were incubated at $37^{\circ} \mathrm{C}$ for 30 minutes in dark and washed with PBS as described above.

The wells of each antigen slide were mounted by $10 \mu \mathrm{l}$ of glycerol and covered by cover slide. Examinations of the stained slides were carried out using immune fluorescent microscope (Germany). The fluorescent lamb was first switched on and allowed to warm up for 10 minutes before the slide were examined under $\mathrm{x} 40$ objective.

Data were analyzed using statistical package for social sciences (SPSS) version 2.3. The differences between variables were checked using the Chi-Square test and ANOVA test, the probability value less than 0.05 was considered significant.

\section{Result}

Out of the 150 samples, 9 (6\% [95\% CI士1.4]) were positive in the blood smear showing Theileria spp. piroplasms, 3 sheep out of 61 (4.9\%) and 6 goats out of 89 (6.7\%) (Table 1).

With regards to animal species, in sheep, the prevalence of Theileria spp. piroplasms was higher in males $2(6.5 \%)$ than in females $1(3.3 \%)$. According to sheep age groups, the highest prevalence of Theileria spp. piroplasm was 3(8.6\%) in age more than twoyear- old and no piroplasm was not detected in less than one years old and 1-2 years old (Table 2). In goats, the prevalence of Theileria spp. piroplasm was higher in females $5(8.3 \%)$, than in males $1(3.4 \%)$. According to goat age groups, the highest prevalence of Theileria spp. piroplasm was $2(10 \%)$ among $1-2$ years old and 3(6.8\%) in more than 2 years old (Table 2 ).

Using indirect fluorescent antibody test (IFAT), the overall prevalence rate of Theileria lestoquardi antibodies was 81(54\% [95\% CI 1.3 ]) (Table 1), 25 sheep out of $61(41 \%)$ and 56 goats out of 89 (62.9\%) (Figure 2).

Concerning animal species, in sheep, the highest prevalence rate of Theileria lestoquardi antibodies was $16(51.6 \%)$ in males, while the lowest rate was $9(30 \%)$ in females (Table 2). With regards to sheep age groups, the highest prevalence rate was $17(48.6 \%)$ in age group more than 2 years, followed by $8(40 \%)$ in less than 1 year, and no antibodies was detected in age group between 1-2 years old (Table 2). In goat, the 
Table 1: Prevalence of Theileria lestoquardi infection of sheep and goats in different locations in Nyala, South Darfur State August and September 2015 using two diagnostic techniques.

\begin{tabular}{|c|c|c|c|}
\hline Location & $\begin{array}{c}\text { Number of sheep and Goats } \\
\text { examined }\end{array}$ & \multicolumn{2}{|c|}{$\begin{array}{c}\text { Diagnostic techniques } \\
\text { BS }\end{array}$} \\
\hline Elseriaf & 20 & $(\% \pm 95 \% \mathrm{CI})$ & $\begin{array}{c}\text { IFAT } \\
(\% \pm 95 \% \mathrm{CI})\end{array}$ \\
\hline Elmauashi & 30 & $1(3.3 \pm 0.36)$ & $10(50 \pm 4.4)$ \\
\hline Mogfgenena & 29 & $1(3.4 \pm 0.36)$ & $15(51.7 \pm 5.5)$ \\
\hline Elshabia & 21 & $0(0.0)$ & $12(57 \pm 5.1)$ \\
\hline Mosy & 30 & $4(13.3 \pm 1.4)$ & $17(56.7 \pm 6.1)$ \\
\hline Slaughter house & 20 & $3(15 \pm 1.3)$ & $13(65 \pm 5.7)$ \\
\hline Total & 150 & $9(6 \pm 1.4)$ & $81(54 \pm 13)$ \\
\hline
\end{tabular}

BS = Blood Smear; IFAT =Indirect fluorescent Antibody Test.

Table 2: Prevalence of Theileria lestoquardi infection in sheep and goats according to sex and age groups in Nyala, South Darfur State during August and September 2015 using two diagnostic techniques.

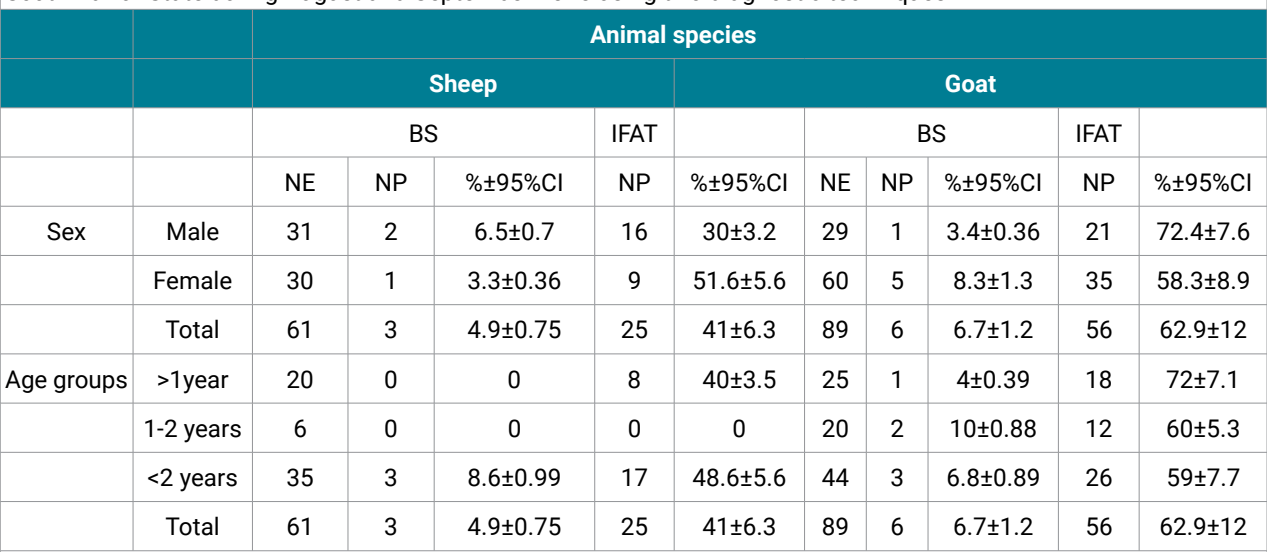

BS = Blood Smear; IFAT =Indirect fluorescent Antibody Test; NE= Number examines; NP=Number positive; \%= percentage.
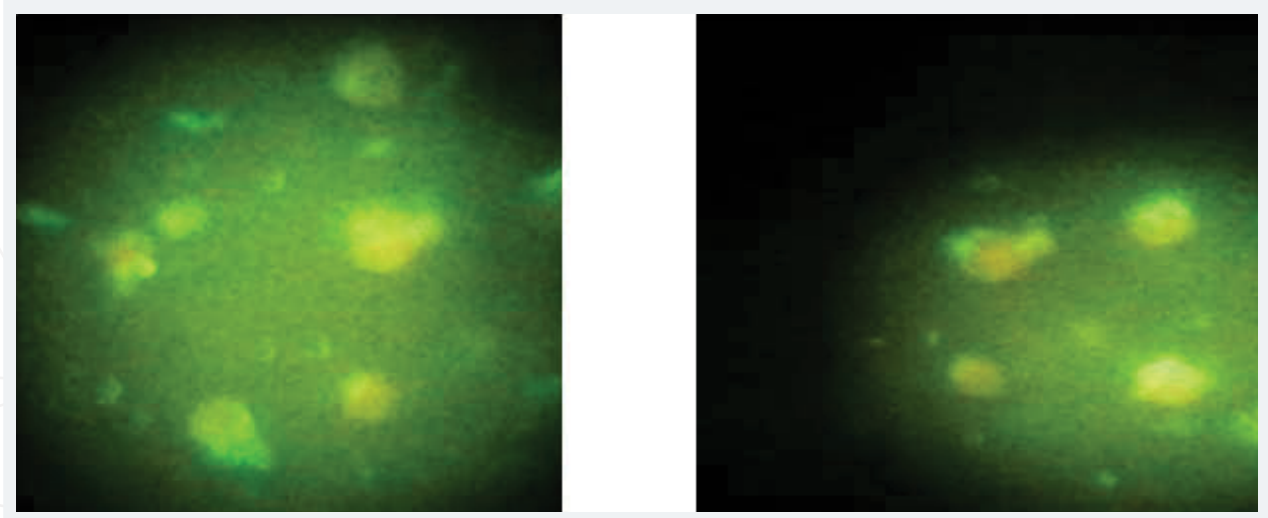

Figure 2: Detection of Theileria lestoquardi antibodies using schizont antigen in IFA test.

highest prevalence rate of Theileria lestoquardi antibodies was 21(72.4\%) in males, while the lowest rate was 35(58.3\%) in females (Table 2). With regard to goat age groups, the highest prevalence rate of Theileria lestoquardi antibodies was 18(72\%) in less than one year, followed by $12(60 \%)$ in age group between 1-2 years old, while the lowest prevalence rate was $26(59 \%)$ in age group more than 2 years old (Table 2 ).

In this study, sheep and goats were found to be infested with two tick genera Amblyomma and Rhipicephalus. Four species of tick were identified: Rhipicephalus evertsi evertsi 16(66.7\%), Amblyomma lepidum 4(16.7\%), Rhipicephalus sanguineus 3(12.5\%), Amblyomma variegatum 1(4.2\%) (Table 3). 
Table 3: Number and percentage of tick species infested sheep and goats in Nyala, South Darfur State during August and September 2015.

\begin{tabular}{|c|c|c|c|}
\hline Tick species & \multicolumn{2}{|c|}{ Number of ticks } & \% of tick \\
\hline Rhipicephalus e. evertsi & Female & Male & 66.7 \\
\hline Amblyomma lepidum & 7 & 9 & 16.7 \\
\hline Rhipicephalus sanguineus & 4 & 0 & 12.5 \\
\hline Amblyomma variegatum & 2 & 1 & 4.2 \\
\hline Total & 1 & 0 & $100 \%$ \\
\hline
\end{tabular}

\section{Discussion}

Tick-bone diseases are widespread causing substantial economic losses and are a constant threat to the development of animal wealth. The previous surveys on tick in the Sudan was conducted by Hoogstraal [10,14-17], major ecological changes have occurred due to extensive animal movement, deforestation, desertification and establishment of large mechanized agricultural schemes [18]. These factors have certainly affected distribution of tick and tick-borne diseases in the Sudan.

The impact of ticks and tick-borne diseases (T and TBDs) in South Darfur State is tremendous due to high abundance of ticks throughout different climatic zones of the region [19]. Recently, due to progressively development of South Darfur State and rapid growing of human population in Nyala town, there is an increase demand for milk and meat products. However, $\mathrm{T}$ and TBDs are the main threat to small ruminants in most locations in Nyala town. There is no epidemiological data on T. lestoquardi infection infecting sheep and goats in Nyala. The present study shed some lights on the epidemiological situation of T. lestoquardi infection in the region using both parasitological and serological tools. The sensitivity of blood smear examination in detection of Theileria parasites in the sampled animals was low and only 9 out of 150 blood smears were positive for Theileria spp. piroplasms. El Hussein et al. [20] had showed the prevalence of $12.2 \%$ in Atbara, North Sudan, while the prevalence rate of Theileria piroplasm in sheep was reported in Khartoum state to be 68\% [21]. Detection of piroplasms microscopically requires high parasitaemia level and well trained personnel. Moreover, the piroplasm detected in blood smears were not necessarily indication of infection with $T$. lestoquardi but could also be other Theileria species.

The seropositivity for T. lestoquardi antibodies was 54\%, using IFAT. The prevalence is higher than previously reported by Salih et al. [7], who was report $10 \%$ seropositive for T. lestoquardi using IFA test from Darfur State. Absence of clinical malignant theileriosis in goats may be attributed to their natural resistance to the parasite, or it may be referred to their resistance to the vector itself. However, Taha et al. [8] had reported an outbreak of malignant ovine theileriosis among goats reared in a mixed flock with sheep in Atbara town, showing clinical signs of malignant ovine theileriosis as well as in the postmortem findings. In this study the seroprevalence of T. lestoquardi in goats was $62.9 \%$, the high prevalence rate may be due to movement of animals in and out Nyala town, it is also possible that due to the introduction of the vector H. anatolicum with the exotic breeds of cattle. The mixed grazing of cattle and small ruminants could affect the spread of the disease among small ruminants. In Khartoum State, an overall prevalence of $20.6 \%$ in sheep and $19.5 \%$ in goats $(19.5 \%)$ was reported using IFAT [22].

The high prevalence of T. lestoquardi in goats was also reported in China [23]. The results indicated higher susceptibility of older groups of sheep and goats to the Theileria infection more than younger one. This finding is in agreement with Darghouth et al. [24], who stated that young animals are less infested because of a low attachment of ticks and the old animals become more tolerant as a result of repeated infestation. 
The prevalence rate of T. lestoquardi infection in female goats was higher than males; this result may be due to the stress of pregnancy [25].

In this study, two genera and four tick species infesting sheep and goats were reported in Nyala. An earlier study in Darfur revealed that twenty tick species in the same region on domestic animals under nomadic systems [26]. Abdallah [19] recorded 15 tick species from sedentary and semi sedentary cattle in South Darfur State, and Gaafar [27] recorded 13 species from semi-intensive system and extensive systems around Nyala town. The current study revealed only two tick species in Nyala town. The lower infestation rate may be attributed to grazing fields, which are relatively open and dry with less vegetation cover. The disappearance of any tick species in any given period is not meaning absence of these species from the area, it may be appear when the optimum climatic conditions prevail [15].

The study concluded that the malignant ovine theileriosis is endemic in Nyala town. H. anatolicum, the efficient vector of ovine theileriosis established in Nyala and probably in other towns of the Darfur [19]. It is suspected that introduction of Friesian breeds of cattle from Central Sudan where H. anatolicum is highly abundant is the source of this tick species in the region. More details studies including a wider area and more advance techniques such as PCR are recommended to further elucidate the epidemiological aspect of MOT in the region.

\section{References}

1. Makala LH, Mangani P, Fujisaki K, Nagasawa $H$. The current status of major tick borne diseases in Zambia. Vet Res. 2003; 34: 27-45. Ref.: https://goo.gl/75ujK1

2. Dolan TT. Theileriosis: a comprehensive review. Rev Sci Tech off Int Epizoot. 1989; 8: 11-36. Ref.: https://goo.gl/Lpkzut

3. Smith MC, Sherman DM. Theileriosis in Goat Medicine. $2^{\text {nd }}(E d)$, Wiley-Blackwell Ames Lowa. USA 2011.

4. Mason FE. Annual report (1914) of Vet Service, Miistry of Agriculture. Veterinary and Pathological Report. Egypt. 1915.

5. Tageldin MH, Zakia AM, Nagwa EG, El Sawi SAS. An outbreak of theileriosis in sheep in Sudan. Trop Anim Health Prod. 1992; 24: 15-16. Ref.: https://goo.gl/yMRQXQ

6. El Hussein AM, El Ghali AA, Mohammed SA. Efficacy of buparvaquone in the treatment of malignant theileriosis of sheep in Ed-Damer Province Nile State Sudan: a field trial. Sud J Vet Res. 1993; 12: 51-57.

7. Salih DA, El Hussein AM, Hayat MF, Taha KM. Survey of Theileria lestoquardi antibodies among Sudanese sheep. Vet Parasitol. 2003; 111: 361-367. Ref.: https://goo.gl/rB6Ckd

8. Taha KM, Salih DA, Ahmed MB, Enan KA, Ali AM, et al. First confirmed report of outbreak of malignant ovine theileriosis among goats in Sudan. Parasitol Res. 2011; 109: 1525-1527. Ref.: https://goo.gl/2x87xH

9. Anon. Ministry of Animal Resources and fishes Sudan Government. Statistical Bulletin for Animal Resources. Issue No. 14. 2004.

10. Hoogstraal H. African Ixodoidea. Vol I. Ticks of the Sudan (with special reference to Equatoria Province and with preliminary reviews of the genera Boophilus, Margaropus and Hyalomma). Department of Medical Zoology. US. Naval Medical Research Unit No. 3. USA. 1956; 1101. Ref.: https://goo.gl/AMLU88

11. Walker AR, Bouattour A, Camicas JI, Horak IG, Latif AA, et al. Ticks of Domestic Animals in Africa: a guide to identification of species. Edinburgh EH10. UK. 2003. Ref.: https://goo.gl/Vr65Ko

12. Burridge MJ, Brown CGD, Kimber CD. Theileria annulata: Cross-reactions between a cell culture schizont antigen of east African species in the indirect fluorescent antibody test. Exp Parasitol. 1974; 35: 374-380. Ref.: https://goo.gl/VZF4YL

13. FAO. Ticks and tick-borne disease control. A Practical Field Manual. Vol. I and II. Food and Agriculture Organization, Rome, Italy. 1984. 
14. Karrar M, Kaiser MN, Hoogstraal H. Ecology and host-relationships of ticks (Ixodoidea) infesting domestic animals in kassala province, Sudan, with special reference to Amblyomma lepidum Donitz. Bull Ent Res. 1963; 54: 509-522. Ref.: https://goo.gl/RNExFQ

15. Osman OM, Ibrahim AM, Osman AG. Some observations on ticks of sedentary cattle in southern Darfur Province. Proc $8^{\text {th }}$ Vet Ass Conf. 1977; 82-92. Ref.: https://goo.gl/q9MPBi

16. FAO. Ticks and ticks-borne Diseases control. The Sudan: Tick Ecology and Tick control. Technical report No. I, AG: CP/SUD/024/DEN, Rome. 1983.

17. Jongejan F, Zivkovic D, Pegram RG, Tatchell RJ, Fison T, et al. Ticks (Acari: Ixodidae) of the Blue and White Nile ecosystems in the Sudan with particular reference to the Rhipicephalus sanguineus group. Exp Appl Acarol. 1987; 3: 331-346. Ref.: https://goo.gl/2Rmeen

18. Hassan SM, Salih DA. Factors affecting geographical distribution of livestock ticks in the Sudan. Sokoto J Vet Sci. 2003; 11: 1-9.

19. Abdallah MM. Studies on tick and tick-borne disease on cattle in South Darfur State of Sudan. M.V.Sc. Thesis U. of K. 2007.

20. El Hussein AM, El Ghali AA, Mohammed SA. Experimental infection of goats with pathogenic ovine Theileria hirci in Ed-Damer Province Sudan. Sudan J Vet Sci Anim Husb. 1998; 37: 190-192.

21. Nagwa ZG. A survey of sheep piroplasmosis in Khartoum Province (Sudan). 1986.

22. Sara AH. Prevalence of Ticks (Acari: Ixodidae) and Theileria lestoquardi Antibodies in Sheep and goats in Khartoum State. Sudan. M.V.Sc. Thesis U of K. 2015.

23. Guo S. Mu Y, Liu Z, Ma D, Yang S, et al. Serological investigation of ovine theileriosis by ELISA in Gannan Tibet Region of Gansu Province in China. Parasitol Research. 2007; 101:197-200. Ref.: https://goo.gl/ADQMS4

24. Darghouth MA, Bouattour A, Ben Miled L, Kilani M, Brown CGD. Epidemiology of tropical theileriosis (Theileria annulata infection of cattle) in an endemic region of Tunisia: characterization of endemicity states. Vet Parasitol. 1996; 65: 199-211. Ref.: https://goo.gl/ZBUkZq

25. Mohammed GM. Molecular, serological and parasitological survey of Thrileria annulata in North Kordofan State, Sudan. M.V.Sc. Thesis U of K. 2012.

26. Osman OM. Preliminary notes on the distribution of ticks (Acarina Ixodidae) in Darfur Province Sudan. Bull. Anim HIth Prod Afr. 1978; 26: 329-333. Ref.: https://goo.gl/v5U1UQ

27. Gaafar BM. Epidemiology of tropical theileriosis in South Darfur State of Sudan. M. V. Sc. Thesis U of K. 2008. 